Article

\title{
Efficient Routines for Obtaining Radiation View-Factor for Non-Uniform Horizons
}

\author{
Tariq Muneer ${ }^{1}$ (D) and Stoyanka Ivanova ${ }^{2, *(D)}$ \\ 1 School of Engineering and Built Environment, Edinburgh Napier University, Edinburgh EH10 5DT, UK; \\ T.Muneer@Napier.ac.uk \\ 2 Civil Engineering and Geodesy, University of Architecture, 1164 Sofia, Bulgaria \\ * Correspondence: solaria@mail.bg
}

Received: 29 March 2020; Accepted: 13 May 2020; Published: 18 May 2020

\begin{abstract}
There are a large number of engineering applications wherein estimation of radiation exchange between surfaces is required. One important part of the latter procedure is to obtain the so-called 'view factor' of the emitter-receiver combination. The principal aim of this article is to present finite-element based procedures to obtain view factor with good time efficiency. As such routines presented here compute the view factor between a non-uniform emitter field and the receiving surface. An example of this is ground-reflected solar radiation to thermal or photovoltaic collectors, the foreground itself being composed of surfaces that have wide-ranging reflectivities. Four routines have been presented here: one based on a uniform grid for emitting and receiving surfaces using a brute force approach (Uniform Populous Grid (UPG)), and another that used non-uniform grid for the receiving surface, where cells' sizes increased in arithmetic progression as one withdraws from the common edge (Non Uniform Grid Populous (NUGP)). The last two routines used combinations of the first two approaches with Monte-Carlo approach (Uniform Grid Monte-Carlo (UGMC) and Non Uniform Grid Monte-Carlo (NUGMC)). It was found that the NUGP algorithm was the most efficient to reduce the calculation error for the same number of computations, it was about 450 times ( 430 for non-uniform reflectivity) more accurate than UPG, 160 (125) times more than UGMC and 70 (60) times more than NUGMC. Finally a comparison of advantages and disadvantages of all four considered routines was added, using the following criteria: ease of programming, computational execution time, accuracy of results obtained, and predictability of the errors.
\end{abstract}

Keywords: view factor; reflected radiation; finite element analysis; solar energy

\section{Introduction}

A number of engineering applications require estimation of radiation exchange between surfaces which in turn leads to computation of 'view factor'. View factor $(\mathrm{VF}), \mathrm{F}_{\mathrm{i}-\mathrm{j}}$ may be defined as the fraction of the radiation leaving surface $i$ that is intercepted by surface $j[1]$.

The view factor (VF) is also known in engineering literature as geometry-, angle-, shape- or configuration factor. This article will be based on obtaining numerical solution for view factor using four procedures. The procedures range from the simplest routine that uses a uniform grid (brute force approach) to a procedure that efficiently combines the Monte-Carlo technique with generation of a non-uniform grid that increases the cell size as one draws away from the emitter-receiver common edge. The manner in which the non-uniform grid is generated has been refined after trialling very many procedures.

A number of engineering applications of the present work are also identified in the present article. 


\section{Case Study Description}

Attention is drawn towards Figures 1 and 2 which demonstrate some of commonly encountered applications of the present work.
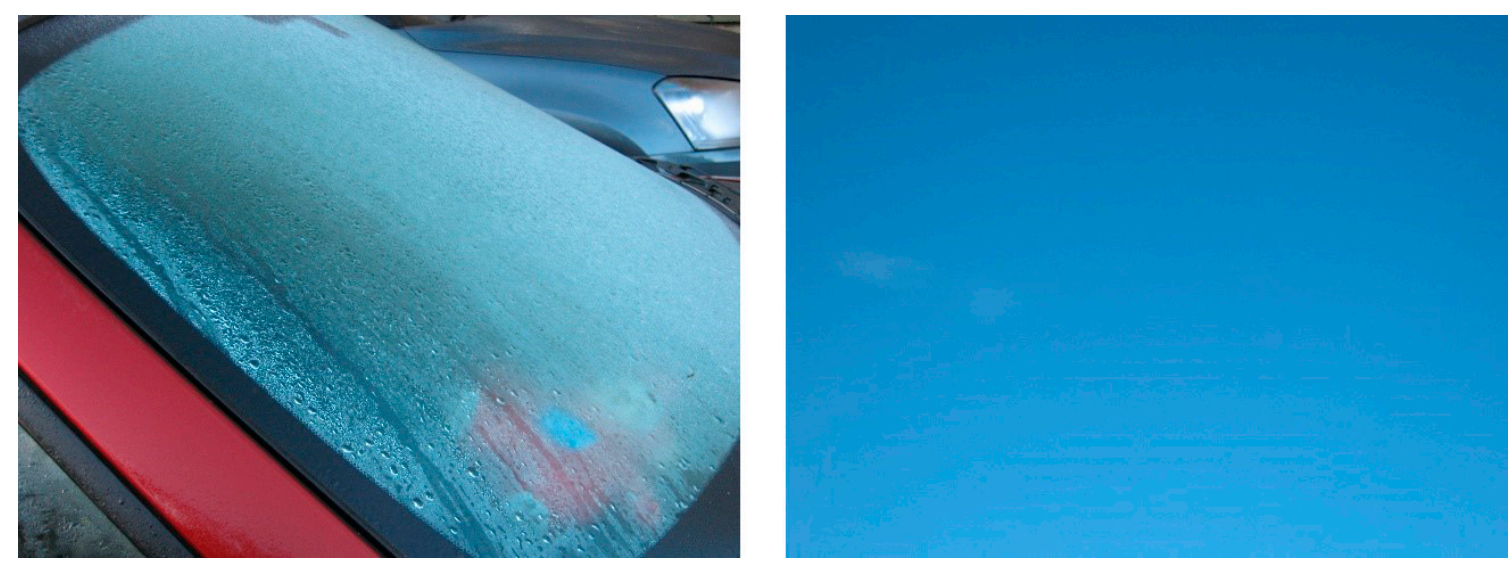

(a)
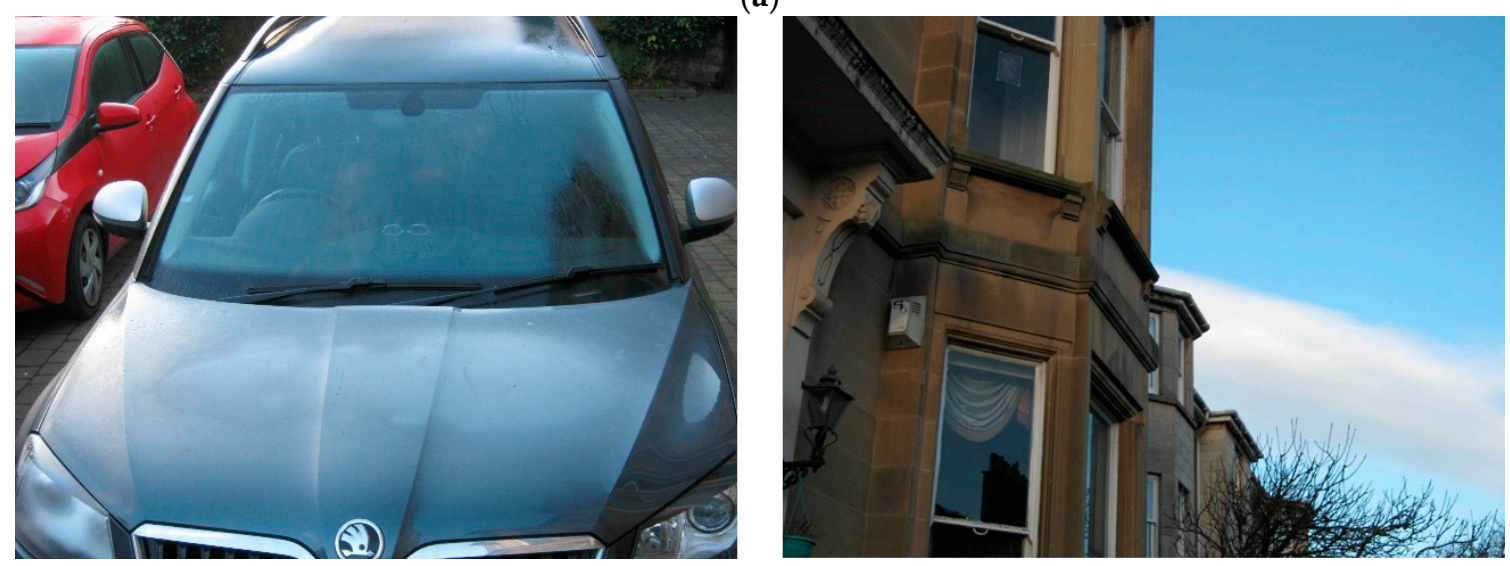

(b)

Figure 1. Pictures that demonstrate the temperature difference of car windscreens that have different objects in their view. The top windscreen (a) acquiring a much lower temperature owing to its view factor that includes only sky. The lower windscreen $(\mathbf{b})$ view is dominated by building surfaces that have a much higher temperature. Note: clear sky at night acquires a high absorptivity and a much lower temperature.

Figure 1 shows the influence that thermal radiation heat transfer has in determining the surface temperature due to overnight cooling. Two automobiles that were parked adjacent to each other may have widely different windshield temperatures purely as a result of the surfaces they have in their view. The convective heat loss is the same for the two windshields as are the view factors. However, the car shown in Figure 1a achieved a much lower surface temperature owing to its radiation emission to a clear sky. The other windshield of Figure $1 \mathrm{~b}$ exchanges thermal radiation, mostly with building surfaces which are much warmer and hence does not show any trace of frost.

Figure 2 shows some aspects of Edinburgh Napier University Building-Integrated Photovoltaic (BIPV) installation. The PV modules face south-east and are inclined at an angle of $75^{\circ}$ from the horizontal. The foreground view is shown in part (b) of Figure 2. As would be expected of an urban setting the foreground is non-uniform and is composed of surfaces with widely different reflectivity. In winter, in particular, the rooftops of the foreground houses are covered with snow which offers a high reflectance. In summer, autumn, and spring the grass and tree tops offer different reflectance. Reference [2] provides more details of the above facility. 


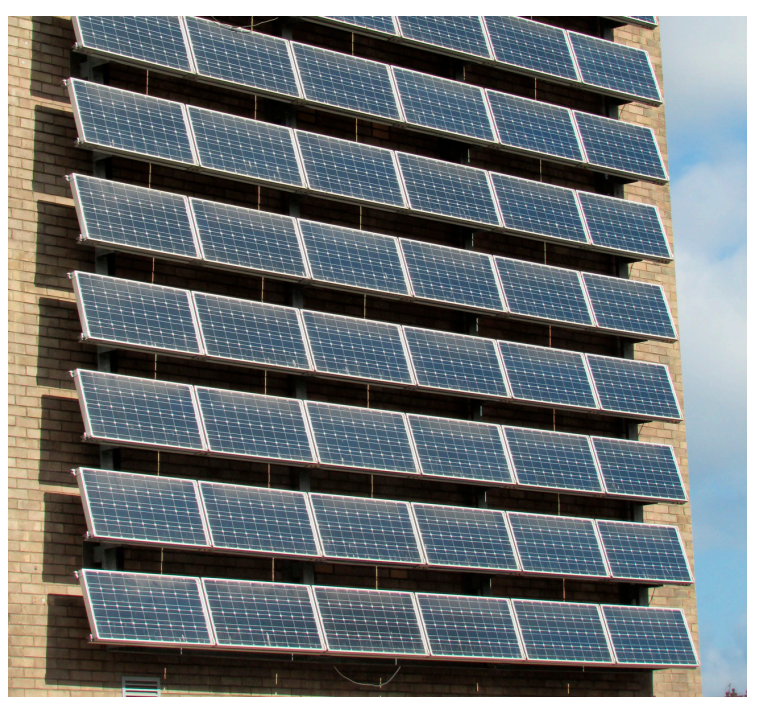

(a)

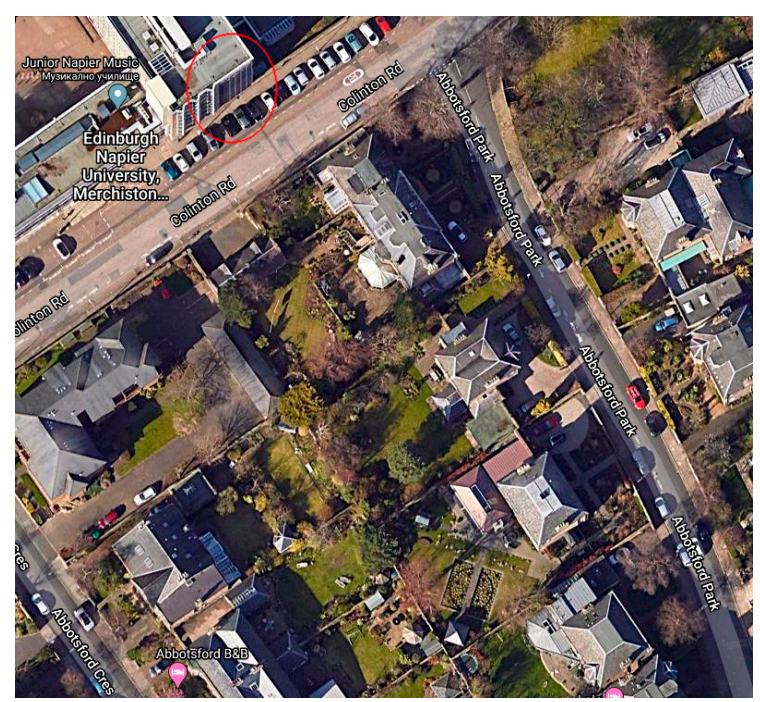

(b)

Figure 2. Edinburgh Napier University (ENU) Building-Integrated Photovoltaic (BIPV) installation: (a) street view of BIPV installation and (b) plan view of ENU-BIPV: roof area $=3766 \mathrm{~m}^{2}$, road area $=3041$ $\mathrm{m}^{2}$, and grass/tree-top area $=7885 \mathrm{~m}^{2}$.

The total incident radiation on a surface such as a PV panel or a solar thermal collector (vertical or inclined) is the sum of beam, sky-diffuse, and ground reflected radiation from various surfaces. It is the latter component that is the subject of this article.

The solar water heating technology has matured over the past several decades. However, the Building Integrated Photovoltaic [BIPV and PV farms have only recently seen a sharp rise in their installations. Furthermore, recent articles have emerged that show the potential of large irradiation gains that may be made by deploying highly reflective surfaces in the foreground of PV modules. Furthermore, the recent advent of bifacial PV modules has brought to attention the importance of ground-reflected radiation. In such a module the PV cells are mounted on both sides of the module, the back side receiving only radiation that is reflected off the ground. The back-side of the PV module has a much higher view factor and hence its estimation gains further importance.

Within the past two decades the cell phone has become an essential tool for communication. The microwave radiation effects of a cell phone may be classified as thermal and non-thermal. The work of Girish Kumar [3] has shown that the above non-thermal effects are several times more harmful than thermal effects. One way to measure the effect of microwave radiation from cell phones is in terms of Specific Absorption Rate (SAR) which is expressed in W/kg. In the US, the SAR limit for cell phones is $1.6 \mathrm{~W} / \mathrm{kg}$. It has therefore been recommended that a cell phone should not be used for more than 18 to $24 \mathrm{~min}$ a day. One cell phone manufacturer has also recommended that the device should be kept at least $25 \mathrm{~mm}$ from the human body. Note that for any scenario study for the use of cell phone view factor between the emitter (cell phone) and human head (receiver) would be required and this would depend on the distance at which the device is held by its user.

In the present article, we present efficient routines for view factor analysis taking into account the non-homogeneous nature of the foreground that may offer a range of varying reflectance.

\section{Procedure for Obtaining View Factor between Two Plane, Inclined Surfaces}

For any two elemental surfaces such as those shown in Figure 3, $\mathrm{F}_{1-2}$ is given as Equation (1):

$$
F_{1-2}=\frac{1}{A_{1}} \int_{A_{1}} \int_{A_{2}} \frac{\cos \Phi_{1} \cos \Phi_{2}}{\pi R_{1-2}{ }^{2}} d A_{2} d A_{1},
$$


where $R_{1-2}$ is the distance between both differential elements $d A_{1}$ and $d A_{2} ; A_{1}$ and $A_{2}$ are the area of the two surfaces; and $\Phi_{1}$ and $\Phi_{2}$ are the angles between the normal vectors to both differential elements and the line between their centres.

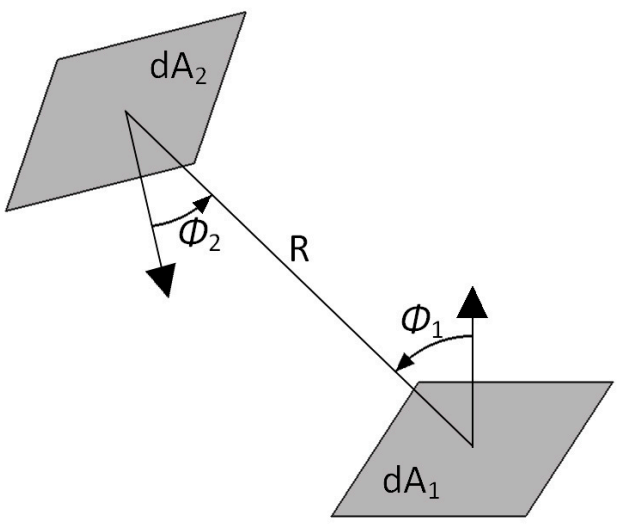

Figure 3. Defining geometry for configuration factor.

See Figure 4. If we apply Equation (1) to two rectangular surfaces $A_{1}$ with dimensions $a \times b$ and $A_{2}$ with dimensions $c \times b$, with angle $\Phi$ between them, then $\beta=\pi-\phi, \cos \Phi_{1}=x_{2} \sin \beta / R$ and $\cos \Phi_{2}=x_{1} \sin \beta / R$ and $R=\sqrt{x_{1}{ }^{2}+x_{2}{ }^{2}+2 x_{1} x_{2} \cos \beta+\left(y_{1}-y_{2}\right)^{2}}$

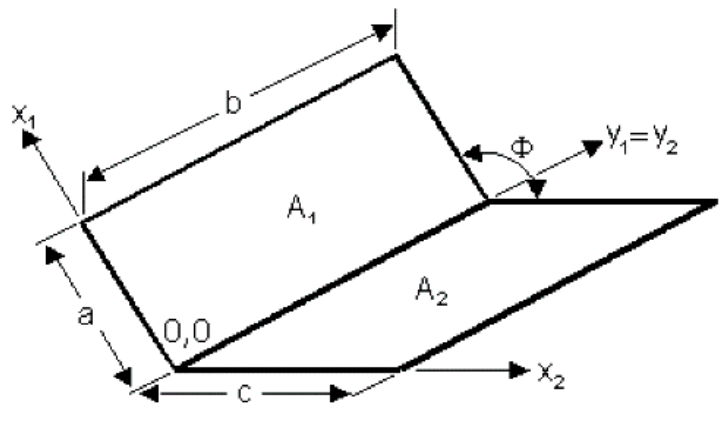

(a)

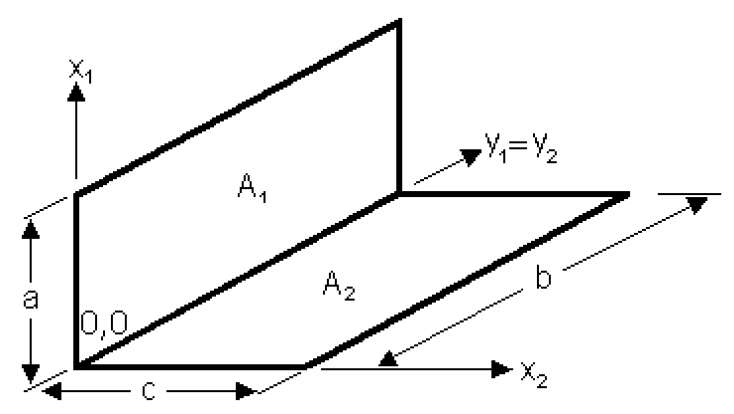

(c)

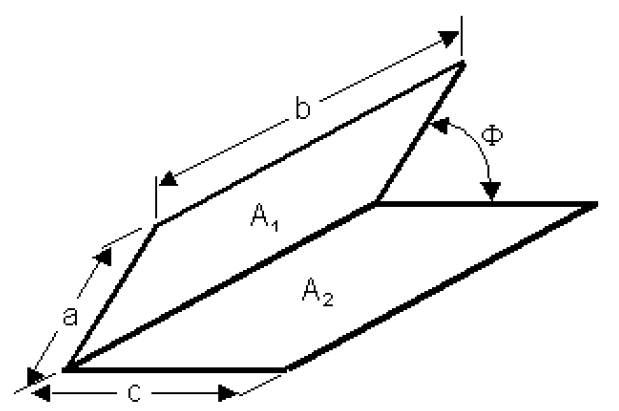

(b)

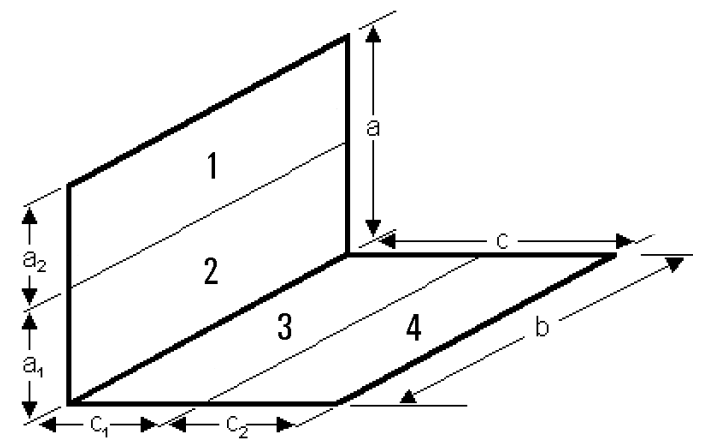

(d)

Figure 4. Two plane surfaces separated by an angle $\Phi$ : (a) co-ordinate system; (b) $\Phi<90^{\circ}$; (c) $\Phi=90^{\circ}$; and (d) $\Phi=90^{\circ}$, split areas.

The resulting integral is Equation (2):

$$
F_{1-2}=\frac{1}{a b} \int_{x_{1}=0}^{a} \int_{y_{1}=0}^{b} \int_{x_{2}=0}^{c} \int_{y_{2}=0}^{b} \frac{x_{1} x_{2} \sin ^{2} \beta}{\pi\left[x_{1}^{2}+x_{2}^{2}+2 x_{1} x_{2} \cos \beta+\left(y_{1}-y_{2}\right)^{2}\right]^{2}} d y_{2} d x_{2} d y_{1} d x_{1},
$$


The solution of this integral is Equation (3), where $A=c / b, B=a / b, C=A^{2}+B^{2}-2 A B \cos \Phi$, and $D=\sqrt{1+A^{2} \sin ^{2} \Phi}[4]$.

$$
\begin{aligned}
& F_{1-2}=-\frac{\sin 2 \Phi}{4 \pi B}\left[A B \sin \Phi+\left(\frac{\pi}{2}-\Phi\right)\left(A^{2}+B^{2}\right)+B^{2} \tan ^{-1}\left(\frac{A-B \cos \Phi}{B \sin \Phi}\right)+A^{2} \tan ^{-1}\left(\frac{B-A \cos \Phi}{A \sin \Phi}\right)\right] \\
& +\frac{\sin ^{2} \Phi}{4 \pi B}\left\{\left(\frac{2}{\sin ^{2} \Phi}-1\right) \ln \left[\frac{\left(1+A^{2}\right)\left(1+B^{2}\right)}{1+C}\right]+B^{2} \ln \left[\frac{B^{2}(1+C)}{C\left(1+B^{2}\right)}\right]+A^{2} \ln \left[\frac{A^{2}\left(1+A^{2}\right)^{\cos 2 \Phi}}{C(1+C)^{\cos 2 \Phi}}\right]\right\} \\
& +\frac{1}{\pi} \tan ^{-1}\left(\frac{1}{B}\right)+\frac{A}{\pi B} \tan ^{-1}\left(\frac{1}{A}\right)-\frac{\sqrt{C}}{\pi B} \tan ^{-1}\left(\frac{1}{\sqrt{C}}\right) \\
& +\frac{\sin \Phi \sin 2 \Phi}{2 \pi B} A D\left[\tan ^{-1}\left(\frac{A \cos \Phi}{D}\right)+\tan ^{-1}\left(\frac{B-A \cos \Phi}{D}\right)\right] \\
& +\frac{\cos \Phi}{\pi B} \int_{0}^{B} \sqrt{1+z^{2} \sin ^{2} \Phi}\left[\tan ^{-1}\left(\frac{z \cos \Phi}{\sqrt{1+z^{2} \sin ^{2} \Phi}}\right)+\tan ^{-1}\left(\frac{A-z \cos \Phi}{\sqrt{1+z^{2} \sin ^{2} \Phi}}\right)\right] d z
\end{aligned}
$$

Thus, Equation (3) still remains unresolved analytically as its very end part requires further integration. The view factor $F_{1-2}$ can be estimated partially analytically, partially numerically.

In a previous work [4], the present authors presented a numerical solution of Equation (2) that enables estimation of view factor for geometries such as those shown in Figure 5.

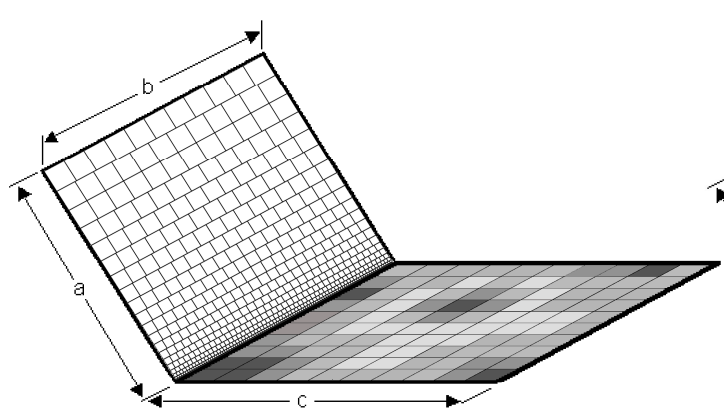

(a)

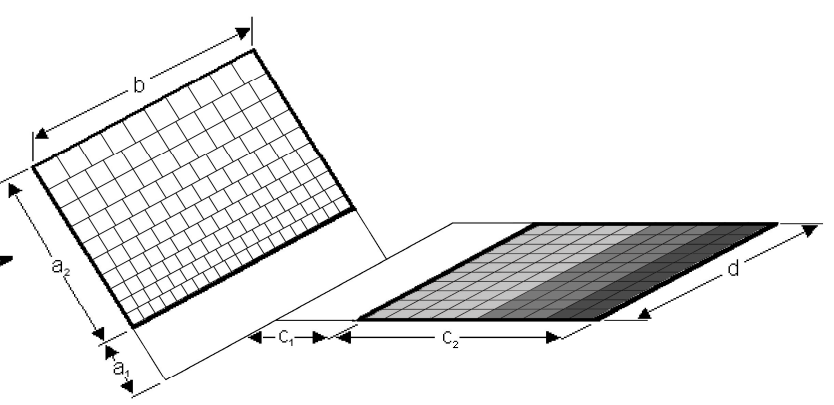

(b)

Figure 5. Case with a non-uniform reflecting surface: (a) surfaces have a common edge; (b) surfaces are non-intersecting. Note that the cells may have different reflectance as depicted by blank, grey, and blackened cells.

Furthermore, a Microsoft Visual Basic for Application (VBA) code was developed that enables the user to obtain the view factor for any geometry and reflectivities for the foreground (surface $A_{2}$ ).

If we consider the rectangular surfaces $A_{\mathrm{i}}$ and $A_{\mathrm{j}}$ with a common edge $b$ as composed of many very small rectangular areas (Figure 5a), numeric integration could be used to solve it where $\mathrm{Na}, \mathrm{Nb}$; and $N c, N d$ are cell numbers for the receiving and emitting surfaces. The finite increments $\Delta a$ through to $\Delta d$ are defined thus: $\Delta u=u / N u$, where $u$ may take the value of $a, b, c$, or $d$. Further details are provided in Reference [4].

$$
F_{j-i}=\frac{\sin ^{2} \Phi}{\pi \cdot N a \cdot N b} \sum_{j_{1}=1}^{N a} \sum_{j_{2}=1}^{N b} \sum_{i_{1}=1}^{N c} \sum_{i_{2}=1}^{N b} \frac{x_{i} x_{j}}{\left[x_{i}{ }^{2}+x_{j}{ }^{2}-2 x_{i} x_{j} \cos \Phi+\left(y_{i}-y_{j}\right)^{2}\right]^{2}} \Delta c \Delta b,
$$

In the case of a receiving surface such as a solar thermal or PV module being irradiated by a field containing patches of differing reflectance (see Figure 5), Equation (4) transforms to Equation (5) thus:

$$
F^{\prime}{ }_{j-i}=\frac{\sin ^{2} \Phi}{\pi a b} \sum_{j_{1}=1}^{N a} \sum_{j_{2}=1}^{N b} \sum_{i_{1}=1}^{N c} \sum_{i_{2}=1}^{N d} \frac{x_{i} x_{j} \rho_{i}}{\left[x_{i}{ }^{2}+x_{j}{ }^{2}-2 x_{i} x_{j} \cos \Phi+\left(y_{i}-y_{j}\right)^{2}\right]^{2}} \Delta a \Delta b \Delta c \Delta d,
$$

\section{Previous Work}

This section shall present a brief survey of recent methods to obtain view factor. Towards the end of this section the novelty of present work is highlighted. 
One of the most authoritative references on the subject of determining view factor is the text due to Siegel and Howell [5]. Furthermore, Howell [6] has provided a compendium of formulations for a range of geometries for obtaining view factor.

In the recent past, Gupta at al [7] have provided a brief review of methods for evaluation of view factor.

Dirsken et al. [8] have obtained Sky View Factor (SVF) for urban, meadows, and forests in their study of impact of SVF resolution on an Urban Heat Island.

Zhang et al. [9] undertook a validation study on the positive correlation between SVF and temperature level in vegetation environments and the negative correlation in built-up areas. Their work was based on measurements obtained from 18 sites.

He et al. [10] provide a detailed account of setting up the Monte Carlo method for obtaining view factor for complex geometries. It is of relevance to note that they have used a million number of rays to obtain the view factor between a finite-length cylinder and a rectangle with two edges that were parallel to cylinder axis and of length equal to that of the cylinder.

Sonmez et al. [11] used a ray-casting technique to obtain view factor that may have applications within the solar energy sector such as PV cells. An interesting result presented in their work that has a parallel to the present work is the relationship between the estimation error for view factor and the number of rays.

Ivanova and Muneer [12] extended their numerical view factor solution work [4] to cover parallel surfaces. Note that in most building energy simulation work you need to obtain view factor for perpendicular, inclined, and parallel surfaces and, thus, references $[4,12]$ provide the required solutions. In the remainder of this section a brief review of computer-based procedures for view factor analyses are presented.

The FACET computer code [13] uses area and contour integration for a range of geometries. The VIEW program [14] uses interactive graphics for obtaining view factor while the MONTE routine uses a Monte Carlo procedure [15].

GLAM enables calculation of view factor for axis symmetric geometries [16] while CNVUFAC [17] uses a computer-graphics analogue of the unit sphere method.

Alciatore et al. [18] attempted a closed-form solution of the general three dimensional radiation configuration factor problem using a microcomputer. Jensen [19] has provided a routine for view factor analysis within the TRNSYS software system. 'TRNSYS' stands for Transient Simulation Software.

Emery et al. [20] have provided a comparative study of methods for comparing the diffuse radiation view factors.

Another authoritative reference on the subject of determining view factor is the book "Engineering Heat Transfer" of Suryanarayana [21].

Vujicic et al. [22] have investigated the Monte Carlo method combined with the finite-element (FE) approach for the estimation of view factors in case of uniform emitting or reflectance. Their approach used random points inside the triangular finite elements of the emitting and receiving surfaces.

Note that a web-based calculator is available that uses the previously published work of Feingold [23]. While the general link for configuration factors calculator is: http://www. thermalradiation.net/tablecon.html, the particular link for obtaining view factor for inclined planes that is based on Feingold's derivation is available under Section C, \#16 (two rectangles with one common edge and included angle of $\Phi$ ) at: http://www.thermalradiation.net/calc/sectionc/C-16.html

There are a number of generic procedures that may be used for obtaining view factor some of which are enumerated below. Note that only those methods which have direct bearing on the present work are explained in further detail. Details of other procedures that are enumerated here but not explained further may be obtained from Reference [4].

(a) Direct integration method which attempts to use a numerical solution of Equation (1);

(b) Unit sphere method;

(c) Ray casting method; 
(d) Cross string method;

(e) Algebraic rule and matrix formulation method; and

(f) Monte Carlo Method.

Method (a) is the subject of the present article and therefore explained via Equations (1)-(5). The novelty of the present work is that it provides numerical solution to view factor computation by hybridization of integration method by exploring brute force, Monte-Carlo, and an innovative approach in which non-uniform grid is used. The algorithm to generate the non-uniform grid has been discussed at length.

\section{Presently Developed View Factor Routines}

This method yields our first of four routines and shall henceforth be called Uniform Populous Grid (UPG) owing to the large number of cells that are generated in this procedure. It obviously is the slowest routine to yield results.

We have also developed a hybrid version of Monte Carlo method within the present work and shall therefore be explained furthermore.

The Monte Carlo (MC) method (Method (f) in the above list) uses repeated random sampling to make numerical estimations of unknown parameters. It enables modeling of complex systems where random variables interact. There are very many applications of Monte Carlo methods though they all rely on random number generation to solve deterministic problems. MCs take their name from the famous casinos located in the city of Monte Carlo.

In the traditional version, MCs are used purely as a statistical technique. In the present context, though, a hybrid integration-MC method has been used which is explained thus. We use MC to select cells at a random, i.e., if $10 \%$ of the cells are to be used for obtaining the view factor for infinitesimal cells then only those cells that result from obtaining numbers between zero and 0.1 are chosen, the range of random numbers generated being between zero and 1.0. This modified version of our MC method is henceforth called Uniform Grid Monte-Carlo (UGMC) routine.

Furthermore, two more time-efficient routines are also introduced in this work and they are described below.

The third approach, named Non Uniform Grid Populous (NUGP) is based on the decreasing the value of the radiation exchange with the increasing of distance between the emitting and receiving cells. Thus, this approach uses a non-uniform grid, where the size of receiving cells enlarges in an arithmetic progression with the increasing of their distance to the common edge between the emitting and receiving surfaces. The emitting surface is divided with a regular grid, as the use of an irregular mesh doesn't lead to an improved accuracy [4].

The fourth approach unifies NUGP and MC and is called Non Uniform Grid Monte Carlo (NUGMC). The goal of such conjunction is to combine the advantages of NUGP and MC and to reach higher accuracy and less computing time.

It was shown via Figure 5 that to estimate the reflected solar radiation from foreground surface a numerical approach may be the only way forward due to their varying reflectance. a uniform grid approach is the easiest to handle in which the reflectance of individual cells may be specified. One such scenario is presented in Figure 3.

\subsection{Uniform Populous Grid}

The classical approach is to have a uniform grid for emitter and receiver surfaces. We may call this technique as Uniform Populous Grid (UPG). Another way to describe this procedure is that it is based on brute force as books on optimisation describe. Figure 5 shows the grid generation for such scheme. The general finite-element numerical solution for such an arrangement is presented in Equation (4). The latter equation was developed by the present authors in a set of two articles that comprehensively cover different geometries [5,12]. 


\subsection{Uniform Grid, Monte Carlo}

This case is the same as case 5.1 but now Monte Carlo approach is used wherein the emitting and receiving cells are selected at random. In our routine, the user may select a Computation Reduction Factor (CRF) to save computational time. For example, if a reduction factor of 5 is chosen then the elemental view factor will be evaluated only if the randomly generated number falls between 0 and 0.2 , the range of all numbers generated by our routine being between 0 and 1 . The final view factor will be the average of all the individual cell-based view factors. We call this procedure UGMC approach for Uniform Grid, Monte Carlo.

\subsection{Non Uniform Grid Populous}

We use the non-uniform grid approach for this algorithm. We call this procedure NUGP for Non Uniform Grid Populous approach.

As described in Section 4, this approach uses different sizes of the cells of the receiving surface. They increase in an arithmetic progression as one moves away from the common edge (Figure 6). The most favourable shape of the cells is a square or as close as possible to it. When the receiving surface is adjoining to the emitting surface, the size of cells in the first lowest row is equal to the step in the arithmetic progression (Figure $6 a, b$ ). When both surfaces are not adjoining, the cells in the first row of the receiving surface need to have a bigger size, proportional to its distance from the common line (Figure 6c.d).

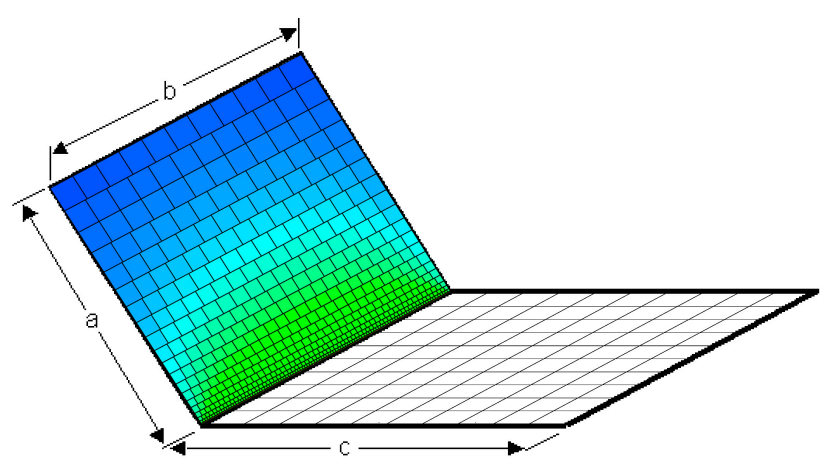

(a)

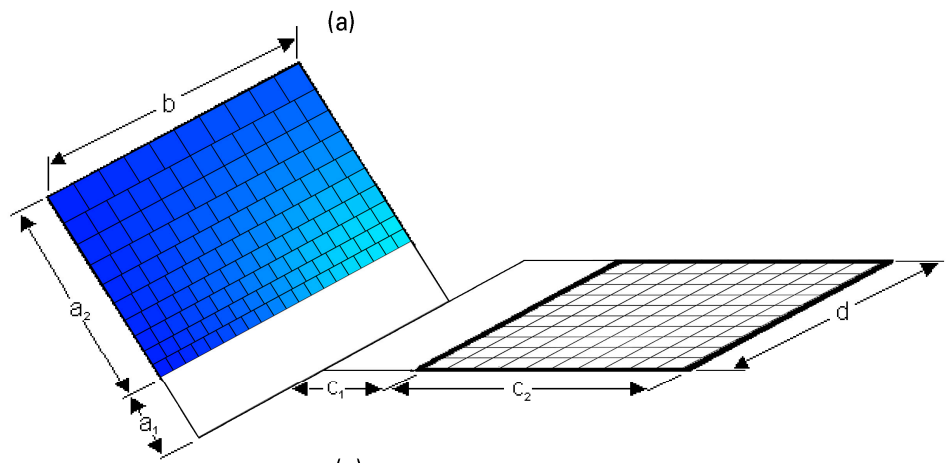

(c)

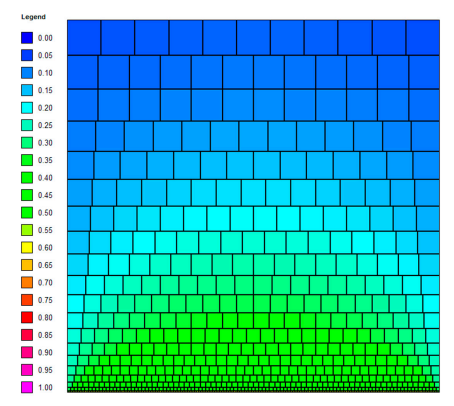

(b)

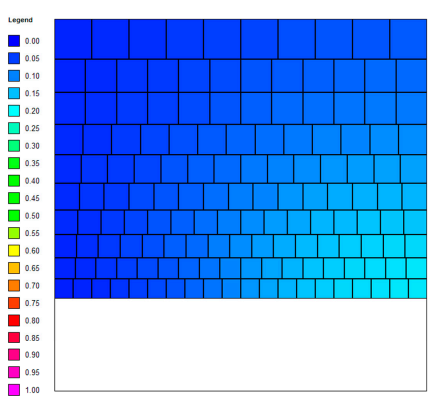

(d)

Figure 6. A non-uniform grid, where cells increase in an arithmetic progression, could be applied on (a) receiving surface with a common edge with the emitting surface; (b) grid for receiving surface with $N_{\mathrm{a}}=20$ rows of cells; (c) receiving surface which is inclined to the emitting surface and they do not share a common edge; and (d) grid for receiving surface with $N_{\mathrm{a}}=10$ rows of cells. The coloured part visualises the VF from the center of each receiving cell to all emitting surface. 
The input value $N_{a}$ is the number of irregular intervals on the side of receiving surface along the axis $x_{1}$ (see Figure $4 a$ ) The number of all square cells on the receiving surface as on Figure $6 \mathrm{~b}$ is given in Equation (6):

$$
N_{\text {receiving_cells }}=\frac{b}{2 a} N_{a}\left(N_{a}+1\right)\left(1+\frac{1}{2}+\frac{1}{3}+\ldots+\frac{1}{N_{a}}\right),
$$

The number of all square cells on the receiving surface as on Figure $6 \mathrm{~d}$ is given in Equation (7):

$$
N_{\text {receiving_cells }}=\frac{b}{2\left(a_{1}+a_{2}\right)}\left(N_{a}+N_{a 0}\right)\left(N_{a}+N_{a 0}+1\right)\left(\frac{1}{N_{a 0}+1}+\frac{1}{N_{a 0}+2}+\ldots+\frac{1}{N_{a 0}+N_{a}}\right)
$$

where $N_{a 0}$ is the number of virtual rows in the interval between the common line of both planes and the lower edge of the receiving surface.

The emitting surface is divided into a uniform grid in $N_{\text {emitting_cells. }}$. The total number of iterations is $N_{\text {receiving_cells. }} . N_{\text {emitting_cells }}$. The experiments with applying such a non-uniform grid on the emitting surface do not show an improvement in the accuracy or reduction of computing time.

The tests with this combined approach with an irregular grid for the receiving surface and uniform grid for emitting surface show better results than the UPG approach presented in Section 5.1. The conclusion is that the larger numbers of cells do not guarantee improved accuracy. It is essential where the grid is more close-meshed and in what degree in comparison with other parts of the surface.

\subsection{Non Uniform Grid Monte Carlo}

We use procedure 5.3 but now select cells, once again using Monte Carlo as described above. We call this NUGMC (for Non Uniform Grid Monte Carlo) approach.

As the emitting surface is divided in a uniform mesh, it is very easy to add Monte Carlo method to this approach. As in 5.2, for each combination of emitting and receiving cell a random number between 0 and 1 is generated, and if it is more than $1 / C R F$, than the VF between both cells is not included in the calculations. This reduces the computing time about CRF times.

One specific feature of this approach is that for every computer run the computed View Factor (VF) varies, because of the impact of the random values. Thus in such study it is important to know the highest positive and negative deviation in per cent from the expected result and the MAPE (Mean Absolute Percent Error), estimated with Equation (8), where $H_{\mathrm{C}}$ is the calculated result, $H_{\mathrm{A}}$ is the analytic result and $n$ is number of tests.

$$
M A P E=\frac{100 \%}{n} \sum \frac{\left|H_{C}-H_{\mathrm{A}}\right|}{H_{\mathrm{A}}}
$$

\section{Results and Discussion}

\subsection{Results for UPG and UGMC}

Tables 1-3 present results obtained by the application of the first two routines that have been presently developed. In each case, the angle between the two planes is 90 degree. Tables 1 and 2 compare the performance of UPG and UGMC routines. In Table 1, we see that as we decreased the cell size from $0.01 \times 0.01$ to $0.002 \times 0.002$ we decreased the error in estimating view factor from 1.588 to $0.318 \%$. An alternate way of exploring this is to take the ratio of the longest side $(0.6 \mathrm{~m})$ to the cell length for the first case (0.01), that ratio is 60 . As we increased that ratio to $300(=0.6 / 0.002)$, we began to see a significant reduction in the estimation error. However, we achieved that at a considerable increase in computational time, i.e., from 4 to $2771 \mathrm{~s}$ for one desktop PC. Note that the computational time will change for different computing machines but the ratio of computational times will be of similar order. The Computational Reduction Factor (CRF) for Table 1 is 1 , as UPG was employed. 
Table 1. Computation of view factor using UPG routine. Example with perpendicular, whole surfaces, uniform reflectance. Routine: Uniform Populous Grid, UPG *.

\begin{tabular}{ccccc}
\hline delX & Error, $\%$ & Time Taken, $\mathbf{s}$ & CRF & No. of Computations \\
\hline 0.01 & 1.588 & 4 & 1 & $2.70 \times 10^{6}$ \\
0.005 & 0.795 & 73 & 1 & $4.32 \times 10^{7}$ \\
0.004 & 0.636 & 182 & 1 & $1.05 \times 10^{8}$ \\
0.002 & 0.318 & 2771 & 1 & $1.69 \times 10^{9}$ \\
\hline
\end{tabular}

${ }^{*}$ Refer to Figure 4c. Dimensions: $a=0.5, b=0.3, c=0.6, \Phi=90^{\circ}$, analytic result $F_{1-2}=0.170278444$.

Table 2. Computation of view factor using UGMC routine. Example with perpendicular, whole surfaces, uniform reflectance. Routine: Uniform Grid Monte-Carlo, UGMC *.

\begin{tabular}{ccccc}
\hline delX & Error, $\%$ & Time Taken, $\mathbf{~}$ & CRF & No. of Computations \\
\hline 0.01 & 1.422 & 2 & 2 & $1.35 \times 10^{6}$ \\
0.005 & 1.260 & 39 & 2 & $2.16 \times 10^{7}$ \\
0.004 & 0.618 & 95 & 2 & $5.27 \times 10^{8}$ \\
0.002 & 0.309 & 1484 & 2 & $8.44 \times 10^{8}$ \\
0.002 & 0.326 & 808 & 4 & $4.22 \times 10^{8}$ \\
0.002 & 0.032 & 470 & 8 & $2.11 \times 10^{8}$ \\
0.002 & 0.219 & 348 & 10 & $1.69 \times 10^{8}$ \\
\hline
\end{tabular}

${ }^{*}$ Refer to Figure 4c. Dimensions: $a=0.5, b=0.3, c=0.6, \Phi=90^{\circ}$, analytic result $F_{1-2}=0.170278444$.

Table 3. Computation of view factor $F_{1-4}$ using UPG and UGMC routines. Example with perpendicular, split surfaces ${ }^{*}$, uniform reflectance.

\begin{tabular}{cccccc}
\hline Approach & delX & Error, $\%$ & No. of Computations & Time Taken, s & CRF \\
\hline UPG & 0.005 & -0.001 & $3.46 \times 10^{7}$ & 58 & 1 \\
UPG & 0.004 & 0.002 & $8.84 \times 10^{7}$ & 141 & 1 \\
UGMC & 0.002 & -0.009 & $1.35 \times 10^{7}$ & 23 & 100 \\
UGMC & 0.001 & 0.005 & $2.70 \times 10^{7}$ & 46 & 800
\end{tabular}

** Refer to Figure 4d. Dimensions: $a=0.5, a_{1}=0.2, \mathrm{~b}=0.6, c=0.3, c_{1}=0.1, \Phi=90^{\circ}$, analytic result $F_{1-4}=0.0678298$.

The CRF varies in Table 2 as we deploy the hybrid UGMC routine as described in Section 5.2. Table 2 shows those results. Now we see the merits of the above hybrid routine, i.e., as we increase the CRF from 1 to 10, the number of computations reduces by the same factor. Note that for a cell size of $0.002 \mathrm{~m}$ and an increase of CRF from 1 to 10 , the computations drop from 1.69 billion to 0.17 billion. Note that there is no change in accuracy even for a CRF of 10 proving the robustness of MC techniques. Similar results were reported by Vujicic et al. [22], even if they used MC method in different way, working with random points inside the finite elements of receiving and emitting surfaces.

Table 3 shows the results for split surfaces, i.e., only a part of surface $A_{1}$ is in view of surface $A_{2}$. This is where the full potential of numerical routines such as those presented here come into play. Methods based on using classical graphical procedures such as those presented in heat transfer texts will rely on view factor algebra for such analysis. For example, with reference to Figure $4 \mathrm{~d}$, view factor $F_{1-4}$ for the split areas using graphical method will be obtained from Equation (9) in a long and tedious way, thus:

$$
F_{1-4}=1+\frac{1}{A_{1}}\left[A_{12} F_{12-34}-A_{1} F_{1-3}-A_{2} F_{2-3}-A_{2} F_{2-4}\right]
$$

In comparison, with numerical integration only the lower and upper limits are changed with Equation (4) thus making the entire operation easily manageable. The efficiency of UGMC is also demonstrated, once again, in Table 3.

Table 4 shows results for inclined surfaces. The exact solution obtained from Feingold's method [21] is used here for validation. The error is estimated using the analytic result. We see that if high accuracy is required then even with UGMC routine a significantly large computer time is required as shown 
in Table 4. Thus, with a cell size of $0.001 \mathrm{~m}$ it is possible to achieve a $99.9 \%$ accuracy but in return a very large computer time is required even with a CRF of 100. Note that for the smallest mesh size shown in Table 4, i.e., delX $=0.001 \mathrm{~m}$ the required computations for a CRF $=1$ exceed 160 billion which would need exorbitant amount of computation time. However, with the CRF of 100 shown in Table 4 the computations drop to a much more manageable figure of 1.6 billion, yet the accuracy remains very similar.

Table 4. Computation of view factor, $F_{12-34}$ using UPG and UGMC routine, uniform reflectance. (Figure $4 \mathrm{a}, \mathrm{b}$ refers).

\begin{tabular}{cccccccccc}
\hline Approach & delX & Error, $\%$ & CRF & $\begin{array}{c}\text { No. of } \\
\text { Computation, s }\end{array}$ & Time Taken, s & $\boldsymbol{\Phi}$ & L * & N * Feingold ** & $\begin{array}{c}\text { Analytic } \\
\text { Result }\end{array}$ \\
\hline UPG & 0.005 & 0.407 & 1 & $2.56 \times 10^{8}$ & 441 & 135 & 0.4 & 0.4 & 0.062773 \\
UGMC & 0.002 & 0.157 & 10 & $1.00 \times 10^{9}$ & 2368 & 135 & 0.4 & 0.4 & 0.062773 \\
UGMC & 0.002 & 0.266 & 20 & $5.00 \times 10^{8}$ & 1573 & 135 & 0.4 & 0.4 & 0.06277322 \\
UGMC & 0.001 & 0.124 & 100 & $1.60 \times 10^{9}$ & 6346 & 135 & 0.4 & 0.4 & 0.062773 \\
UPG & 0.005 & 2.836 & 1 & $2.56 \times 10^{8}$ & 443 & 45 & 0.4 & 0.4 & 0.06277322 \\
UGMC & 0.002 & 1.465 & 10 & $1.00 \times 109$ & 2427 & 45 & 0.4 & 0.4 & 0.56016 \\
UGMC & 0.002 & 0.748 & 20 & $5.00 \times 108$ & 1712 & 45 & 0.4 & 0.4 & 0.56277322 \\
UGMC & 0.002 & 1.207 & 20 & $5.00 \times 108$ & 1760 & 45 & 0.4 & 0.4 & 0.56015973 \\
\hline
\end{tabular}

${ }^{\prime *} \mathrm{~L}=\mathrm{c} / \mathrm{b}, \mathrm{N}=\mathrm{a} / \mathrm{b} .{ }^{~} * *$ The particular link for obtaining view factor for inclined planes that is based on Feingold's derivation is available under Section C, \#16 (two rectangles with one common edge and included angle of $\Phi$ ) at: http://www.thermalradiation.net/calc/sectionc/C-16.html.

Monte Carlo approach uses random numbers and, thus, the results of the computation of VF vary (see row 3 and row 4 in Table 4, where the error of the computed VF for the same case is different).

To study the dispersion of the estimated VF values, they are computed 1000 times for the same example (Figure $4 \mathrm{c}, a=0.5, c=0.6, b=0.3, \mathrm{CRF}=9, \Phi=90^{\circ}, \mathrm{delX}=0.01$, No. of computations $=3.0 \times 10^{5}$ ), the exact analytic result is 0.17028 .

The estimated $1000 \mathrm{VF}$ values with UGMC approach are sorted from smallest to biggest. The calculated negative and positive errors in percent (also sorted) are illustrated in Figure 7a. They vary from $-3.4 \%$ to $+9.34 \%$. Then the interval between the minimum and maximum error is divided in 20 parts and for each part the associated errors are counted. That statistic is used to create the chart presented in Figure $7 \mathrm{~b}$, which is a histogram of the errors.

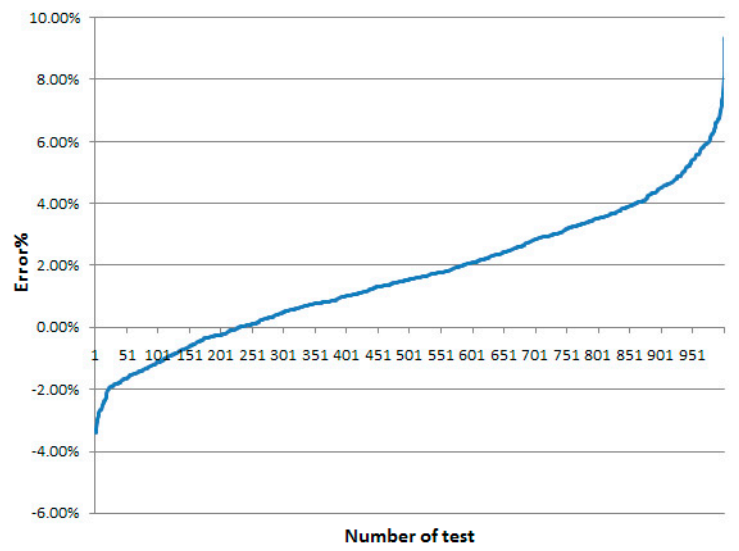

(a)

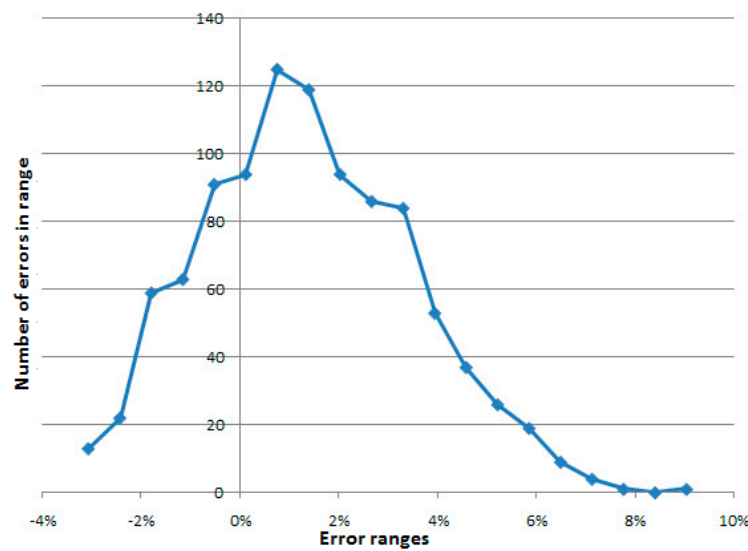

(b)

Figure 7. Analysis of the errors with UGMC for the case in Figure 4c after 1000 tests: (a) calculated and sorted errors and (b) histogram of those errors.

\subsection{Results for NUGP and NUGMC}

Table 5 shows results for inclined surfaces with NUGP approach. Again the exact solution obtained from Feingold's method [21] and analytical integration results are used here for validation. For this approach delX cannot be defined for the receiving surface, because the size of the cells vary 
increasing from the common edge to the opposite edge. Thus, the table has a column delX for the emitting surface and a column $\mathrm{Na}$ for the receiving surface. The CRF factor is 1, because all cells of the emitting surface participate in the calculations.

Table 5. Computation of view factor using NUGP routine. Example with perpendicular, whole surfaces, uniform reflectance. Routine: Non Uniform Grid Populous, NUGP*.

\begin{tabular}{cccccc}
\hline Na & delX & Error, $\%$ & Time Taken, s & CRF & No. of Computations \\
\hline 50 & 0.01 & 0.016 & 10 & 1 & $6.24 \times 10^{6}$ \\
50 & 0.005 & 0.017 & 39 & 1 & $2.50 \times 10^{7}$ \\
50 & 0.004 & 0.017 & 60 & 1 & $3.90 \times 10^{7}$ \\
50 & 0.002 & 0.018 & 240 & 1 & $1.56 \times 10^{8}$ \\
60 & 0.005 & 0.012 & 58 & 1 & $3.72 \times 10^{7}$ \\
70 & 0.004 & 0.009 & 126 & 1 & $8.15 \times 10^{7}$ \\
80 & 0.002 & 0.007 & 674 & 1 & $4.36 \times 10^{8}$ \\
100 & 0.002 & 0.004 & 1131 & $7.09 \times 10^{8}$ \\
\hline \multicolumn{7}{c}{$a=0.5, b=0.3, c=0.6, \Phi=90^{\circ}$, analytic result $F^{\prime} 1-2=0.170278444}$.
\end{tabular}

The NUGMC approach is used for the same case and the results are placed in Table 6. It is like Table 5 (for the same VF), only the value of CRF varies and this has influence on the error, time taken and number of computations. The Monte Carlo approach uses random numbers and thus the results of the computation of VF vary (see row 1 and row 2 in Table 6, where the error of the computed VF for the same case is different). The error is unpredictable, sometime the increase of CRF leads to reduction of time (which is expected) and to a smaller error (which is unanticipated, e.g., see rows 5-8 of Table 6).

Table 6. Computation of view factor using NUGMC routine. Example with perpendicular, whole surfaces, uniform reflectance. Routine: Uniform Grid Monte-Carlo, NUGMC*.

\begin{tabular}{cccccc}
\hline Na & delX & Error, $\%$ & Time Taken, s & CRF & No. of Computations \\
\hline 50 & 0.01 & 0.127 & 5 & 2 & $3.12 \times 10^{6}$ \\
50 & 0.01 & -0.054 & 5 & 2 & $3.12 \times 10^{6}$ \\
60 & 0.005 & 0.024 & 32 & 2 & $1.86 \times 10^{7}$ \\
70 & 0.004 & 0.068 & 71 & 2 & $4.07 \times 10^{7}$ \\
80 & 0.002 & 0.012 & 367 & 2 & $2.18 \times 10^{8}$ \\
80 & 0.002 & -0.004 & 206 & 8 & $1.09 \times 10^{8}$ \\
80 & 0.002 & -0.001 & 125 & 10 & $5.45 \times 10^{7}$ \\
80 & 0.002 & -0.010 & 109 & $4.36 \times 10^{7}$ \\
\hline * Refer to Figure 4c. Dimensions: $a=0.5, b=0 ., c=0.6, \Phi=90^{\circ}$, analytic result $F_{1-2}=0.170278444$
\end{tabular}

As in Section 6.1, two charts are prepared with NUGMC and displayed in Figure 8. First of them is generated using calculated and sorted 1000 errors for the case in Figure $4 \mathrm{c}(a=0.5, c=0.6$, $b=0.3, \Phi=90^{\circ}, \mathrm{CRF}=9, \mathrm{del} X=0.01, \mathrm{Na}=50$ ), the second image is a histogram of the same errors, which vary from $-1.56 \%$ to $+1.51 \%$. It is clear that the errors display an almost normal distribution (Gaussian curve). 


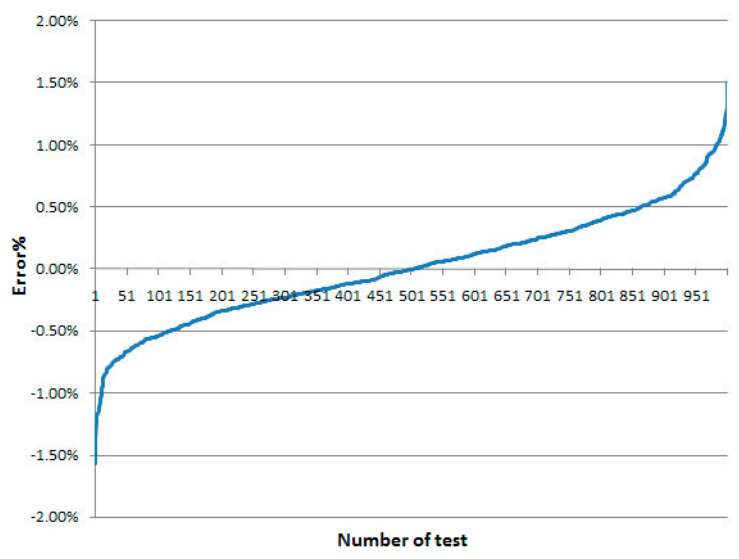

(a)

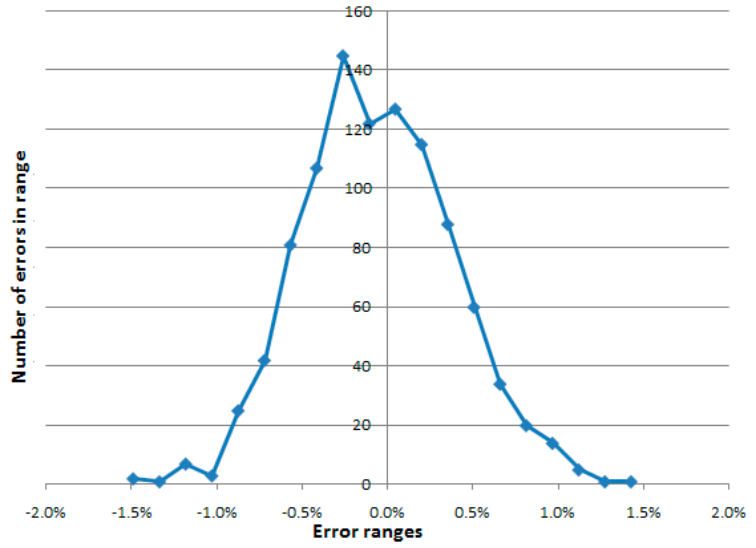

(b)

Figure 8. Analysis of the errors with NUGMC for the case in Figure 4c after 1000 tests: (a) calculated and sorted errors; (b) histogram of those errors.

There exists another way to generate approximately the same number of computations of NUGMC with $\mathrm{CRF}=9\left(6.93 \times 10^{5}\right)$. This is when NUGP is used with cells 3 times bigger than in NUGMC approach, which means that the number of cells is 9 times less. The difference between the two approaches is that with NUGMC the centre of 1 random cell of a cluster of 9 cells is used, and in the case of NUGP the centre of a grid containing 9 cells $(3 \times 3$ cells $)$ is used. The error for the NUGP approach for this example is constantly $0.005 \%$ for the same number of computations, while for NUGMC it varies from $-1.56 \%$ to $+1.51 \%$.

\subsection{Comparison of All Results for Uniform and Non-Uniform Reflectance}

To compare both approaches with implemented Monte Carlo method, two sets of 1000 tests with the same number of computations $\left(7 \times 10^{5}\right)$ are illustrated in Figure 9 . They clearly explain why the NUGMC approach is better than UGMC - the curve of NUGMC errors (in blue) in Figure 9a is closer to 0 , and the error histogram of NUGMC (in blue again) is more symmetrical, more narrow and closer to $0 \%$.

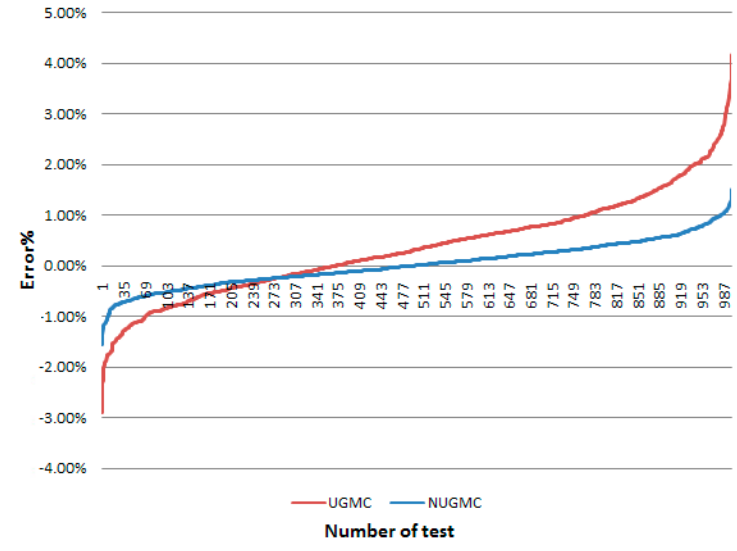

(a)

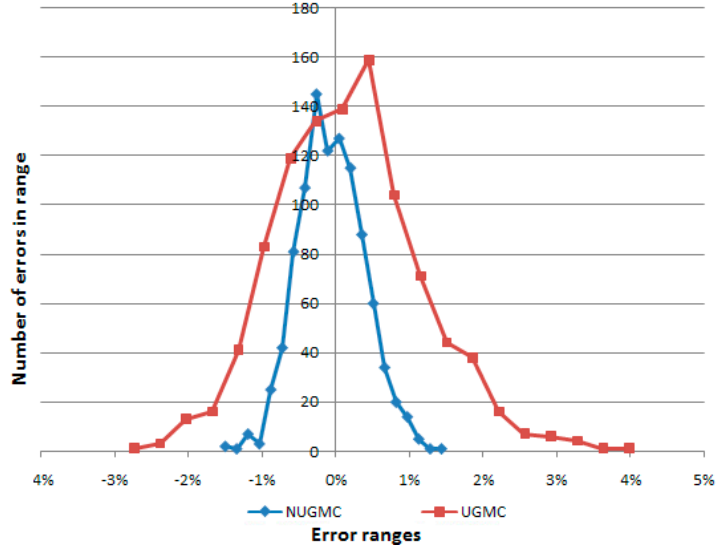

(b)

Figure 9. Comparison of the errors with UGMC and NUGMC for the case in Figure 4c after 1000 tests with the same number of computations $\left(7 \times 10^{5}\right)$ : (a) calculated and sorted errors and $(\mathbf{b})$ histogram of those errors.

Additionally, Table 7 is prepared to compare the performance of the four approaches presented here. It includes results for all approaches for the same case (in Figure 4c) with almost the same number of computations. The smallest error $(0.005 \%)$ belongs to NUGP approach, which is 454 times 
lower than for UPG (2.274\%). The errors for the approaches that use MC method vary, thus they are estimated as MAPE\% according Equation (8) for 1000 tests. The MAPE\% for NUGMC is 2.3 times lower than for UGMC approach.

Table 7. Computation of view factor using all routines. Example with perpendicular, whole surfaces, uniform reflectance, case in Figure $4 c^{*}$.

\begin{tabular}{cccccc}
\hline Approach & CRF & No. of Computations & Time Taken, $\mathbf{s}$ & Error, $\%$ & Error Range, $\%$ \\
\hline UPG & - & $7.04 \times 10^{5}$ & 1 & 2.274 & - \\
UGMC & 9 & $7.04 \times 10^{5}$ & 1 & 0.815 & -2.91 to +4.16 \\
NUGP & - & $6.94 \times 10^{5}$ & 1 & 0.005 & - \\
NUGMC & 9 & $6.93 \times 10^{5}$ & 1 & 0.353 & -1.56 to +1.51 \\
\hline \multicolumn{5}{c}{${ }^{*}$ Dimensions: $a=0.5, b=0.3, c=0.6, \Phi=90^{\circ}$, analytic result $F_{1-2}^{\prime}=0.170278444}$.
\end{tabular}

Next, Table 8 is prepared to compare the performance of the four approaches for the case of non-uniform reflecting surface. It includes results for all approaches for the same case (in Figure 4c) with almost the same number of computations, using Equation (5) instead of Equation (4). The albedo of the horizontal reflecting surface varies from 0.2 to 0.8 (see Figure $10 \mathrm{a})$. The smallest error $(0.006 \%)$ belongs again to NUGP approach, which is 422 times lower than for UPG $(2.531 \%)$. The errors for the approaches with MC method are estimated as MAPE\% according Equation (8) for 1000 tests. The MAPE \% for NUGMC is 2.1 times lower than for UGMC approach. In general for both approaches with MC, the error is unpredictable and varies in large ranges. The grids for NUGP and NUGMC approaches with corresponding colourised VF are displayed in Figure 10b,c. Both coloured grids look similar, but they are not the same because of the randomised calculations in NUGMC.

Table 8. Computation of view factor using all routines. Example with perpendicular, whole surfaces, case in Figure 4c, with non-uniform reflecting horizontal surface.

\begin{tabular}{cccccc}
\hline Approach & CRF & No. of Computations & Time Taken, $\mathbf{s}$ & Error, $\%$ & Error Range, $\%$ \\
\hline UPG & - & $7.04 \times 10^{5}$ & 1 & 2.531 & - \\
UGMC & 9 & $7.04 \times 10^{5}$ & 1 & 0.753 & -2.98 to +3.07 \\
NUGP & - & $6.94 \times 10^{5}$ & 1 & 0.006 & - \\
NUGMC & 9 & $6.94 \times 10^{5}$ & 1 & 0.359 & -1.31 to +1.57 \\
\hline \multicolumn{7}{r}{ " Dimensions: $a=0.5, b=0.3, c=0.6, \Phi=90^{\circ}$, analytic result $\mathrm{F}_{1-2}=0.122269561}$.
\end{tabular}

'* Dimensions: $a=0.5, b=0.3, c=0.6, \Phi=90^{\circ}$, analytic result $\mathrm{F}_{1-2}=0.122269561$.

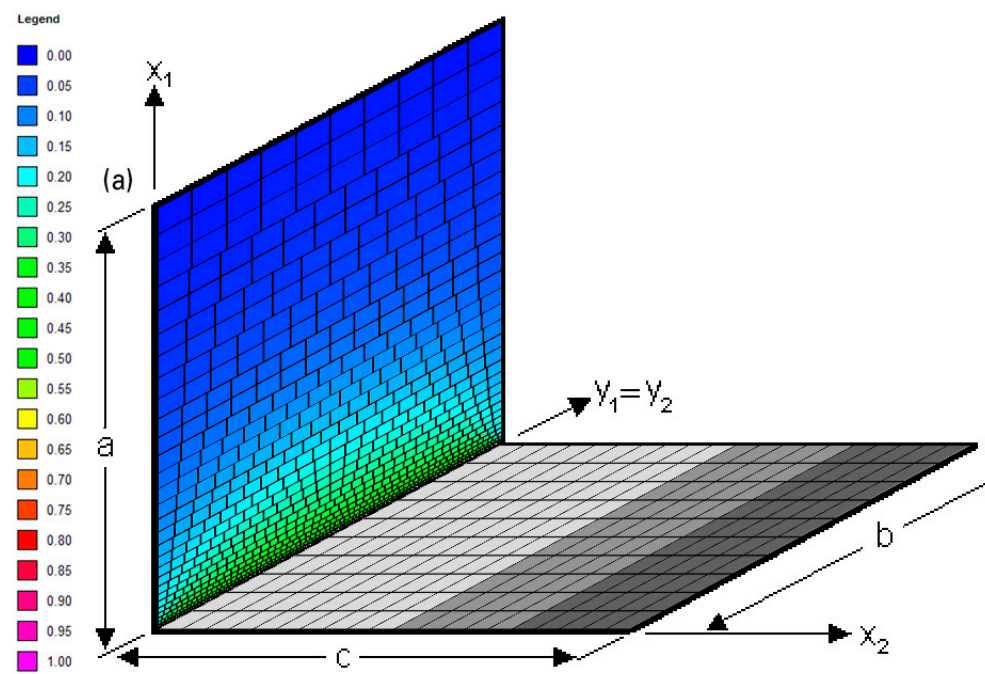

(b)

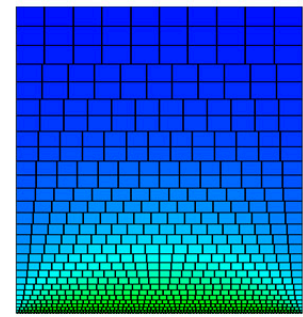

Figure 10. Application of NUGP and NUGMC approaches for the case in Figure 4c. The horizontal surface is non-uniform reflecting, the vertical surface is receiving. The coloured part visualises the VF from the center of each receiving cell to all emitting surface: (a) case with two surfaces with common edge; (b) grid for NUGP; and (c) grid for NUGMC. 
From a practical point of view, if we consider any case of two surfaces exchanging radiant energy, we find that both emitting and receiving surfaces are in majority of cases non-uniform with respect to their radiative properties. In this case, it is difficult to use the classical, calculus-based approach to obtain the view factor. The only procedure left is to use a finite-element based approach in which the surfaces are divided into elements for which the radiative properties may be prescribed individually. That is the chief advantage of all presented techniques. Furthermore, it is important to keep the computational time to a minimum. In this article time-saving, efficient routines are presented that address the above issues.

All four considered approaches could be used for non-uniform emitting and reflecting. These with MC method (UGMC and NUGMC) can be used for a fast evaluation of the energy exchange, when the time saving is desirable. This could allow a fast energy "preview" and comparison of different building designs. It has to be underlined that all these four approaches are not suitable for specular reflecting.

Table 9 include information about the advantages and disadvantages of the four considered approaches, which are compared using the following criteria: ease of programming, computational execution time, accuracy of results obtained, and predictability of the errors.

Table 9. Comparison of advantages and disadvantages of all four considered approaches.

\begin{tabular}{lllll}
\hline & Advantages & Compared to & Disadvantages & Compared to \\
\hline \multirow{2}{*}{ UPG } & Easy programming & UGMC, NUGP & $\begin{array}{l}\text { Low accuracy } \\
\text { Slow execution }\end{array}$ & $\begin{array}{l}\text { NUGP } \\
\text { NUGP, UGMC }\end{array}$ \\
\hline \multirow{2}{*}{ UGMC } & Faster execution & UPG & Lower accuracy & UPG, NUGP \\
& Easy programming & NUGP, NUGMC & Unpredictable error & UPG \\
\hline \multirow{2}{*}{ NUGP } & Higher accuracy & UPG, UGMC & More complex programming & UPG, UGMC \\
& Fast execution & UPG & & More complex programming \\
\multirow{2}{*}{ NUGMC } & Faster execution & NUGP & Unpredictable error & NUGP \\
\hline
\end{tabular}

\section{Conclusions}

This article presents four approaches for the estimation of view factors as a measure of radiation exchange between surfaces. All of them are based on a micromesh, finite-element approach. Broadly speaking two types of geometries were considered-surfaces that were perpendicular to each other, and those that were at an obtuse angle. Two approaches-UPG (Uniform Populous Grid) and UGMC (Uniform Grid Monte-Carlo) use regular mesh for both emitting and receiving surface; the other two-NUGP (Non Uniform Grid Populous) and NUGMC (Non Uniform Grid Monte Carlo) use non-regular mesh for receiving surface only, the emitting surface is divided into a uniform grid. Two approaches (UGMC and NUGMC) use a hybrid Monte Carlo method to decrease the number of computations, the hybrid method resulting from the present work. Between these two approaches the following conclusions may be drawn: using the Monte-Carlo approach offers a powerful tool to reduce the number of computations without compromising the error. In one case, for example, with the error around the $0.01 \%$ mark with the Monte-Carlo approach it is possible to reduce the computations from 436 million to 43.6 million without any significant change in accuracy. The respective computer time taken for the execution of those routines was 674 and $109 \mathrm{~s}$. Another remarkable finding of the present research was that owing to its purely random nature the Monte-Carlo method may reduce a significant amount of computational effort while reducing the error at the same time. To give an example for the case when the two surfaces were at an angle of 135 degree the Monte-Carlo approach reduced the error from $0.407 \%$ for uniform grid to $0.124 \%$ while using a Computational Reduction Factor of 100 . So, it is possible to get a double bonus-reduced computations and higher accuracy.

Finally a comparison of advantages and disadvantages of all four considered routines was added, using the following criteria: ease of programming, computational execution time, accuracy of results obtained, and predictability of the errors. 
The order in which the present routines may be arranged with respect to increasing accuracy are UPG followed by UGMC, NUGMC, and NUGP.

Tables 7 and 8 numerically sums up these findings for uniform and non-uniform reflectance, i.e., for the same number of computations the NUGP approach is most accurate, it is about 450 times (430 for non-uniform reflectivity) more accurate than UPG, 160 (125) times more than UGMC, and 70 (60) times more than NUGMC. This demonstrates the potential of the NUGP approach. There is scope for further development of this work.

Author Contributions: Conceptualization, T.M. and S.I.; methodology, T.M.; software, T.M. and S.I.; validation, T.M. and S.I.; formal analysis, T.M. and S.I.; investigation, T.M. and S.I.; resources, T.M.; data curation, T.M. and S.I.; writing-original draft preparation, T.M. and S.I.; writing—review and editing, T.M.; visualization, S.I.; supervision, T.M.; project administration, T.M. All authors have read and agreed to the published version of the manuscript.

Funding: This research received no external funding.

Conflicts of Interest: The authors declare no conflict of interest.

\section{References}

1. Incropera, F.P.; DeWitt, D.P. Fundamentals of Heat and Mass Transfer, 5th ed.; Wiley: New York, NY, USA, 2002.

2. Muneer, T.; Younes, S.; Lambert, N.; Kubie, J. Life cycle assessment of a medium-sized photovoltaic facility at a high latitude location. Proc. Imeche Part a J. Power Energy 2006, 220, 517-524. [CrossRef]

3. Kumar, G. Mobile Tower/mobile phone radiation hazards. Available online: https://www.ee.iitb.ac.in/ $\sim\{\}$ mwave/GK-Cell\%20Tower-\%20Hazard-Sept11.pdf (accessed on 29 January 2020).

4. Muneer, T.; Ivanova, S.M.; Kotak, Y.; Gul, M. Finite-element view-factor computations for radiant energy exchanges. J. Renew. Sustain. Energy 2015, 7, 033108. [CrossRef]

5. Siegel, R.; Howell, J.R. Thermal Radiation Heat Transfer, 4th ed.; Taylor and Francis: New York, NY, USA, 2002.

6. Howell, J.R. A Catalog of Radiation Configuration Factors; McGraw-Hill: New York, NY, USA, 1982.

7. Gupta, M.K.; Bumtariya, K.J.; Shukla, A.J.; Patel, P.; Khan, Z. Methods for evaluation of radiation view factor: a review. Mater. Today Proc. 2017, 4, 1236-1243. [CrossRef]

8. Dirsken, M.; Ronda, R.J.; Theeuwes, N.E.; Pagani, G.A. Sky view factor calculations and its application in urban heat island studies. Urban Clim. 2019, 20, 100498.

9. Zhang, J.; Zhonghua, G.; Lu, Y.; Lin, P. The impact of sky view factor on thermal environments in urban parks in a subtropical coastal city of Australia. Urban For. Urban Green. 2019, 44, 126422. [CrossRef]

10. He, F.; Shi, J.; Zhou, L.; Li, W.; Li, X. Monte Carlo calculation of view factors between some complex surfaces: Rectangular plane and parallel cylinder, rectangular plane and torus, especially cold-rolled strip and W-shaped radiant tube in continuous annealing furnace. Int. J. Therm. Sci. 2018, 134, 465-474. [CrossRef]

11. Sonmnez, F.F.; Ziar, H.; Isabella, O.; Zeman, M. Fast and accurate ray-casting-based view factor estimation method for complex geometries. Sol. Energy Mater. Sol. Cells 2019, 200, 109934. [CrossRef]

12. Ivanova, S.M.; Muneer, T. Finite-element heat-transfer computations for parallel surfaces with uniform or non-uniform emitting. J. Renew. Sustain. Energy 2016, 8, 015102. [CrossRef]

13. Shapiro, A.B. FACET - A Computer View Factor Computer Code for Axisymmetric, 2D Planar and 3D Geometries with Shadowing; UCID-19887; University of California, Lawrence National Laboratory: Livermore, CA, USA, 1983.

14. Emery, A.F. VIEW-A Radiation View Factor Program with Interactive Graphics for Geometry Definition; NASA Computer Software Management and Information Center: Atlanta, GA, USA, 1986.

15. Burns, P.J. MONTE-A Two-Dimensional Radiative Exchange Factor Code; Colorado State University: Fort Collins, CO, USA, 1983.

16. Garelis, E.; Rudy, T.E.; Hickman, R.B. GLAM-A Steady State Numerical Solution to the Vacuum Equation of Transfer in Cylindrically Symmetric Geometries; UCID-19157; University of California, Lawrence National Laboratory: Livermore, CA, USA, 1981.

17. Wong, R.L. User's Manual for CNVUFAC—The General Dynamics Heat Transfer Radiation View Factor Program; UCID-17275; University of California, Lawrence National Laboratory: Livermore, CA, USA, 1976. 
18. Alciatore, D.; Lipp, S.; Janna, W.S. Closed-form solution of the general three dimensional radiation configuration factor problem with microcomputer solution. In Proceedings of the 26th National Heat Transfer Conference, Philadelphia, PA, USA, 6-9 August 1989.

19. Jensen, C.L. Thermal Radiation Analysis System, TRNSYS-II user's manual, ANSI version 1.0; Martin Marietta Aerospace Corp.: Denver, CO, USA, 1987.

20. Emery, A.F.; Johansson, O.; Lobo, M.; Abrous, A. a comparative study of methods for comparing the diffuse radiation view factors for complex structures. J. Heat Transf. 1991, 113, 413-422. [CrossRef]

21. Suryanarayana, N.V. Engineering Heat Transfer.; West Publishing Company: New York, NY, USA, 1995.

22. Vujicic, M.; Lavery, N.P.; Brown, S.G.R. View Factor Calculation Using the Monte Carlo Method and Numerical Sensitivity. Commun. Numer. Methods Eng. 2006, 22, 197-203. [CrossRef]

23. Feingold, A. Radiant interchange configuration factors between various selected plane surfaces. Proc. R. Soc. Lond. Ser. A-Math. Phys. 1966, 292, 51-60.

(C) 2020 by the authors. Licensee MDPI, Basel, Switzerland. This article is an open access article distributed under the terms and conditions of the Creative Commons Attribution (CC BY) license (http://creativecommons.org/licenses/by/4.0/). 